\title{
Lean non-alcoholic fatty liver disease: do not forget diabetes
}

Dimitrios loannis Patoulias ${ }^{1}$, Maria-Styliani Kalogirou²

\author{
${ }^{1}$ First Department of Internal Medicine, General Hospital “Hippokration”, \\ Thessaloniki, Greece \\ ${ }^{2}$ Second Propedeutic Department of Internal Medicine, Aristotle University \\ of Thessaloniki, General Hospital “Hippokration”, Thessaloniki, Greece
}

Submitted: 18 August 2019

Accepted: 10 October 2019

Arch Med Sci Atheroscler Dis 2019; 4: e248-e249

DOI: https://doi.org/10.5114/amsad.2019.90146

Copyright (c) 2019 Termedia \& Banach

\author{
Corresponding author: \\ Dr. Dimitrios loannis \\ Patoulias \\ First Department \\ of Internal Medicine \\ General Hospital \\ "Hippokration" \\ Thessaloniki, Greece \\ Phone: +30 6946900777 \\ E-mail: dipatoulias@gmail. \\ com
}

We have read with great interest the recently published article authored by Li et al. [1]. In their cross-sectional study, the authors demonstrated that elevated fasting plasma glucose was significantly more frequent among overweight/obese patients compared to lean patients with non-alcoholic fatty liver disease (NAFLD) [1].

In another, recently published cross-sectional study enrolling 1,509 patients with NAFLD from an Asian population, it was confirmed that lean NAFLD patients feature a milder metabolic profile compared to overweight and obese NAFLD patients, as estimated by indices including fasting insulin and homeostasis model assessment of insulin resistance (HOMA-IR) [2]. Similar results were obtained from another study enrolling 187 Caucasian subjects, including healthy controls, lean and obese NAFLD patients; however, it was demonstrated that NAFLD patients did not differ regarding their impaired response to oral glucose challenge [3]. Despite the fact that significantly fewer patients with lean NAFLD compared to obese NAFLD suffer from diabetes, it has been shown that patients with lean NAFLD and a medium waist circumference have greater odds for incident diabetes than patients with obese NAFLD and a medium waist circumference, as well [4].

More recent data confirm that lean NAFLD is strongly and positively associated with the development of diabetes. Sung et al. demonstrated that lean NAFLD increases the risk of diabetes by 1.17 times (hazard ratio $(\mathrm{HR})=2.17,95 \% \mathrm{Cl}: 1.56-3.03)$ in men and by 1.86 times in women $(\mathrm{HR}=2.86,95 \% \mathrm{Cl}: 1.50-5.46)$, while corresponding numbers further increase when fatty liver is assessed along with insulin resistance [5]. Sinn et al. have also shown that lean NAFLD patients exhibit an $18 \%$ increase in the risk of incident diabetes compared to lean subjects without NAFLD ( $\mathrm{HR}=1.18,95 \% \mathrm{Cl}: 1.03-1.35)$, suggesting the need for close monitoring of these patients for the potential development of metabolic abnormalities [6].

Fasting plasma glucose appears to be a major indicator for the progression of NAFLD to non-alcoholic steatohepatitis (NASH) in patients with lean NAFLD [7]. In addition, patients with lean NAFLD exhibit greater all-cause and cardiovascular mortality, compared to lean, non-NAFLD subjects, with diabetes arising as a crucial determinant for all-cause mortality ( $\mathrm{HR}=1.35,95 \% \mathrm{Cl}: 1.04-1.75)$ [8]. Vice versa, presence of diabetes at baseline appears to be an independent risk factor for the development of incident lean NAFLD [9]. 
Collectively, it seems that even lean NAFLD patients are at high risk of glucose homeostasis derangement, which may in fact contribute to the progression of the underlying liver disease and the significant increase in related morbidity and mortality. In addition, diabetes represents an additional risk factor for all-cause and cardiovascular mortality for these patients. Thus, this sensitive sub-population warrants a close follow-up, including meticulous assessment of glucose homeostasis, even if the latter is normal at baseline.

\section{Conflict of interest}

The authors declare no conflict of interest.

\section{References}

1. Li H, Chen Y, Tian X, et al. Comparison of clinical characteristics between lean and obese nonalcoholic fatty liver disease in the northeast Chinese population. Arch Med Sci Atheroscler Dis 2019; 4: e191-5.

2. Shao C, Ye J, Li F, Feng S, Wang W, Zhong B. Different predictors of steatosis and fibrosis severity among lean, overweight and obese patients with nonalcoholic fatty liver disease. Dig Liver Dis 2019; 51: 1392-9.

3. Feldman A, Eder SK, Felder TK, et al. Clinical and metabolic characterization of lean caucasian subjects with non-alcoholic fatty liver. Am J Gastroenterol 2017; 112: 102-10.

4. Fracanzani AL, Petta S, Lombardi R, et al. Liver and cardiovascular damage in patients with lean nonalcoholic fatty liver disease, and association with visceral obesity. Clin Gastroenterol Hepatol 2017; 15: 1604-11.e1.

5. Sung KC, Seo DC, Lee SJ, Lee MY, Wild SH, Byrne CD. Non alcoholic fatty liver disease and risk of incident diabetes in subjects who are not obese. Nutr Metab Cardiovasc Dis 2019; 29: 489-95.

6. Sinn DH, Kang D, Cho SJ, et al. Lean non-alcoholic fatty liver disease and development of diabetes: a cohort study. Eur J Endocrinol 2019 Jun 1. pii: EJE-19-0143.R1.

7. Denkmayr L, Feldman A, Stechemesser L, et al. Lean patients with non-alcoholic fatty liver disease have a severe histological phenotype similar to obese patients. J Clin Med 2018; 7: pii: E562.

8. Golabi P, Paik J, Fukui N, Locklear CT, de Avilla L, Younossi ZM. Patients with lean nonalcoholic fatty liver disease are metabolically abnormal and have a higher risk for mortality. Clin Diabetes 2019; 37: 65-72.

9. Niriella MA, Kasturiratne A, Pathmeswaran A, et al. Lean non-alcoholic fatty liver disease (lean NAFLD): characteristics, metabolic outcomes and risk factors from a 7-year prospective, community cohort study from Sri Lanka. Hepatol Int 2019; 13: 314-22. 